\title{
Global Development and Climate Change: A Game Theory Approach
}

\section{Climate Change and Global Development pp 17-35}

\author{
Cite as
}

Chapter

First Online: 09 May 2019

\section{Part of the Contributions to Economics book series (CE)}

\section{Abstract}

The increasing concern with climate change is one of the main issues of our time, and thus we aim to theoretically and mathematically analyse its causes. However our approach follows a different stream of thought, presenting the reasoning and decision-making processes between technical and moral solutions. We have resorted to game theory models in order to demonstrate cooperative and non-cooperative scenarios, ranging from the traditional to the evolutionary within game theory. In doing so we are able to glimpse the development of modern society and a paradigm shift regarding human control over nature and to what extent it is harmful to the sustainability of our environment and the survival of future generations. Merging different fields of knowledge, we present a theoretical-philosophical approach, combined with empirical-mathematical solutions taking into account the agent-based behaviour guided blindly by instrumental rationality.

\section{Keywords}

Evolutionary game theory Global development Game theory

Moral and technical solutions International relations and politics Economics

Sustainable development

The chapter is inspired by a previous paper by Ingo Andrade de Oliveira and Stephánie Rycken. We would also like to thank the extremely constructive comments and suggestions from an anonymous reviewer. 


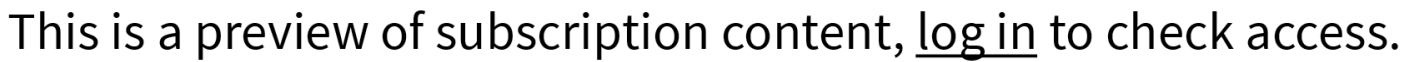

\section{References}

Akerlof, G. A. (1997). Social distance and social decisions. Econometrica, 65, $1005-1027$.

CrossRef (https://doi.org/10.2307/2171877)

Google Scholar (http://scholar.google.com

/scholar_lookup?title=Social\%20distance\%20and\%20social\%2odecisions\& author $=$ GA. $\% 20$ Akerlof\&journal $=$ Econometrica\&volume $=65 \&$

pages=1005-1027\&publication_year $=1997)$

Arthur, B. A., Durlauf, S. N., \& Lane, D. A. (1997). The economy as an evolving complex system II. Reading, MA: Addison-Wesley.

Google Scholar (http://scholar.google.com

/scholar_lookup?title=The\%2oeconomy\%20as\%20an\%2oevolving\%2ocomplex \%20system\%20II\&author=BA.\%20Arthur\&author=SN.\%2oDurlauf\& author=DA.\%20Lane\&publication_year $=1997$ )

Assad, E. D., Buainain, A. M., Pinto, H. S., Rocha De Sousa, M., \& Duarte, V. S. (2013). Climate change: Challenges for Brazil. In D. Vajpeyi (Ed.), Climate change, sustainable development and international security (Chap. 6) (pp. 169-199). Lanham, MD: Lexington (An imprint of Rowman and Littlefield). isbn:978-0-7391-8146-1 https://rowman.com/ISBN/9780739181461 (https://rowman.com/ISBN/9780739181461)

Google Scholar (http://scholar.google.com

/scholar_lookup?title=Climate\%2ochange

\%3A\%20Challenges\%2ofor\%2oBrazil\&author=ED.\%20Assad\& author=AM.\%20Buainain\&author=HS.\%20Pinto\& author=M.\%20Rocha\%20De\%20Sousa\&author=VS.\%20Duarte\& pages $=169-199 \&$ publication_year $=2013)$

Aumann, R. (1959). Acceptable points in general cooperative n-person games. In R. D. Luce \& A. W. Tucker (Eds.), Contributions to the Theory 23 of Games IV, Annals of Mathematics Study (Vol. 40, pp. 287-324). Princeton, NJ: Princeton University Press.

Google Scholar (http://scholar.google.com

/scholar_lookup?title=Acceptable\%2opoints\%20in\%2ogeneral\%2ocooperative \%20n-person\%2ogames\&author=R.\%20Aumann\&pages=287-324\& publication_year=1959)

Auerswald, H., Konrad, K. A., \& Thum, M. (2011). Adaption, mitigation and risk-taking in climate policy (CESifo Working Paper Series 3320). Munich: CESifo Group.

Google Scholar (https://scholar.google.com/scholar?q=Auerswald\%2C\%20H. \%2C\%2oKonrad\%2C\%20K.\%20A.\%2C\%20\%26\%20Thum\%2C\%20M. \%20\%282011\%29.\%20Adaption\%2C\%20mitigation\%20and\%20risktaking\%2oin\%2oclimate\%2opolicy \%20\%28CESifo\%20Working\%20Paper\%20Series\%203320\%29.\%20Munich \%3A\%20CESifo\%20Group.)

Binmore, K. (1994). Playing fair: Game theory and the social contract (Vol. 1). Cambridge, MA: MIT Press.

Google Scholar (http://scholar.google.com 
/scholar_lookup?title=Playing\%2ofair

\%3A\%20Game\%2otheory\%20and\%20the\%20social\%2ocontract\&

author=K.\%2oBinmore\&publication_year=1994）

Binmore, K. G. (1998). Game theory and the social contract: Just playing (Vol. 2). Cambridge, MA: MIT Press.

Google Scholar (http://scholar.google.com

/scholar_lookup?title=Game\%20theory\%20and\%20the\%20social\%20contract

\%3A\%20Just\%2oplaying\&author=KG.\%2oBinmore\&publication_year=1998)

Buchholz, W., \& Sandler, T. (2017). Successful Leadership in Global Public Good Provision: Incorporating Behavioural Approaches. Environmental and

Resource Economics, 67, 591-607.

CrossRef (https://doi.org/10.1007/s10640-016-9997-2)

Google Scholar (http://scholar.google.com

/scholar_lookup?title=Successful\%2oLeadership\%2oin\%2oGlobal\%2oPublic\% 20Good\%20Provision\%3A\%20Incorporating\%20Behavioural\%20Approaches\& author=W.\%20Buchholz\&author=T.\%20Sandler\&

journal=Environmental\%20and\%20Resource\%20Economics\&volume=67\&

pages $=591-607 \&$ publication_year $=2017)$

Bull, H. (2002). The anarchical society (3rd ed.). New York: Palgrave.

Google Scholar (http://scholar.google.com/scholar_lookup?title=\%oA\%20

\%20\%20\%20\%20\%20\%20\%20\%20\%20\%20\%20\%20\%20\%20\%20\%20

\%20\%20\%20The\%20anarchical\%20society\%oA\%20\%20\%20\%20\%20\%20\%20

\%20\%20\%20\%20\%20\%20\%20\%20\%20\%20\%20\&author=H.\%2oBull\&

publication_year=2002)

Decanio, S. J., \& Fremstad, A. (2013). Game theory and climate diplomacy.

Ecological Economics, 85, 177-187.

CrossRef (https://doi.org/10.1016/j.ecolecon.2011.04.016)

Google Scholar (http://scholar.google.com

/scholar_lookup?title=Game\%20theory\%2oand\%2oclimate\%2odiplomacy\& author=SJ.\%20Decanio\&author=A.\%2oFremstad\&

journal=Ecological\%20Economics\&volume $=85$ \&pages $=177-187 \&$

publication_year=2013)

Gardiner, S. M. (2006). A perfect moral storm: Climate change,

intergenerational ethics and the problem of moral corruption. Environmental

Values, 15(3), 397-413.

CrossRef (https://doi.org/10.3197/096327106778226293)

Google Scholar (http://scholar.google.com

/scholar_lookup?title=A\%2operfect\%20moral\%20storm

\%3A\%2oClimate\%20change

\%2C\%2ointergenerational\%2oethics\%20and\%2othe\%2oproblem\%20of\%2omo

ral\%20corruption\&author=SM.\%20Gardiner\&

journal=Environmental\%2oValues\&volume $=15$ \&issue $=3$ \&pages $=397-413 \&$

publication_year=2006)

Goodchild, M. F., Anselin, L., Appelbaum, R. P., \& Harthorn, B. H. (2000).

Toward spatially integrated social science. International Regional Science

Review, 23(2), 139-159.

CrossRef (https://doi.org/10.1177/016001760002300201)

Google Scholar (http://scholar.google.com

/scholar_lookup?title=Toward\%20spatially\%2ointegrated\%20social\%2oscience \&author=MF.\%20Goodchild\&author=L.\%20Anselin\&

author=RP.\%2OAppelbaum\&author=BH.\%20Harthorn\&

journal=International\%20Regional\%20Science\%20Review\&volume=23\&

issue $=2$ \&pages $=139-159$ \&publication_year $=2000$ )

Habermas, J. (1984). The theory of communicative act (T. McCarthy, Trans.). Boston: Beacon Press. 
Google Scholar (https://scholar.google.com/scholar?q=Habermas\%2C\%20J. \%20\%281984\%29.\%20The\%20theory\%20of\%20communicative\%20act \%20\%28T.\%20McCarthy\%2C\%20Trans.\%29.\%20Boston \%3A\%2oBeacon\%2oPress.)

Hardin, G. (1968). The tragedy of the commons. Science, New Series, 162(3859), 243-253.

Google Scholar (http://scholar.google.com /scholar_lookup?title=The\%2otragedy\%20of\%20the\%20commons\& author $=$ G. $\% 20$ Hardin\&journal $=$ Science $\% 2 C \% 20$ New $\% 20$ Series\&volume $=162 \&$ issue $=3859$ \&pages $=243-253 \&$ publication_year $=1968$ )

Harrington, L. J., Frame, D. J., Fischer, E. M., Hawkins, E., Joshi, M., et al. (2016). Poorest countries experience earlier anthropogenic emergence of daily temperature extremes. Environmental Research Letters, 11(5), 055007.

CrossRef (https://doi.org/10.1088/1748-9326/11/5/055007)

Google Scholar (http://scholar.google.com

/scholar_lookup?title=Poorest\%20countries\%20experience\%2oearlier\%20anth ropogenic\%2oemergence\%20of\%20daily\%2otemperature\%2oextremes\& author=LJ.\%20Harrington\&author=DJ.\%20Frame\&author=EM.\%20Fischer\& author=E.\%20Hawkins\&author=M.\%20Joshi\&

journal=Environmental\%20Research\%20Letters\&volume $=11 \&$ issue $=5 \&$ publication_year=2016)

Heal, G. (2017). The economics of the climate. Journal of Economic Literature, 55, 1046-1063.

CrossRef (https://doi.org/10.1257/jel.20151335)

Google Scholar (http://scholar.google.com

/scholar_lookup?title=The\%20economics\%20of\%20the\%2oclimate\& author $=$ G.\%20Heal\&journal=Journal\%20of\%20Economic\%2oLiterature\& volume $=55 \&$ pages $=1046-1063 \&$ publication _year $=2017)$

Howard, N. (1994a). Drama theory and its relation to game theory. Part 1: Dramatic resolution Vs. rational solution. Group Decision and Negotiation, 3, 187-206.

CrossRef (https://doi.org/10.1007/BFo1384354)

Google Scholar (http://scholar.google.com

/scholar_lookup?title=Drama\%20theory\%20and\%20its\%2orelation\%20to\%20 game\%20theory.\%20Part\%201

\%3A\%2oDramatic\%20resolution\%2oVs.\%2orational\%20solution\& author=N.\%2oHoward\&journal=Group\%2oDecision\%20and\%2oNegotiation\& volume $=3 \&$ pages $=187-206 \&$ publication_year $=1994)$

Howard, N. (1994b). Drama theory and its relation to game theory. Part 2: Formal model of the resolution process. Group Decision and Negotiation, 3, 207-235.

CrossRef (https://doi.org/10.1007/BF01384355)

Google Scholar (http://scholar.google.com

/scholar_lookup?title=Drama\%20theory\%20and\%20its\%2orelation\%20to\%20 game\%20theory.\%20Part\%202

\%3A\%2oFormal\%2omodel\%20of\%2othe\%2oresolution\%2oprocess\& author=N.\%2oHoward\&journal=Group\%2oDecision\%20and\%20Negotiation\& volume $=3 \&$ pages $=207-235 \&$ publication_year $=1994)$

Keohane, R. O., \& Victor, D. G. (2016). Cooperation and discord in global climate policy. Nature Climate Change, 6, 570-575.

CrossRef (https://doi.org/10.1038/nclimate2937)

Google Scholar (http://scholar.google.com

/scholar_lookup?title=Cooperation\%20and\%2odiscord\%2oin\%2oglobal\%2ocli mate\%2opolicy\&author=RO.\%20Keohane\&author=DG.\%20Victor\& journal $=$ Nature $\% 20$ Climate $\% 20$ Change $\&$ volume $=6 \&$ pages $=570-575 \&$ 
publication_year=2016)

Khun, H. (1953). Extensive games and the problem of information. In H. W. Kuhn, \& A. W. Tucker (Eds.), Contributions to the theory of games (Vol. II, pp. 193-216). In H. W. Kuhn (Ed.). (1996). Classics in game theory (pp. 46-68). Princeton: Princeton University Press.

Google Scholar (https://scholar.google.com/scholar?q=Khun\%2C\%20H. \%20\%281953\%29.\%20Extensive\%2ogames\%20and\%2othe\%2oproblem\%2oof \%20information.\%2OIn\%2OH.\%2OW.\%2OKuhn\%2C\%2O \%26\%20A.\%2oW.\%20Tucker\%20\%28Eds.\%29\%2C \%20Contributions\%20to\%2othe\%2otheory\%20of\%2ogames\%20\%28Vol.\%20II \%2C\%20pp.\%20193\%E2\%80\%93216\%29.\%20In\%20H.\%2oW.\%2oKuhn \%20\%28Ed.\%29.\%20\%281996\%29.\%20Classics\%20in\%2ogame\%20theory \%20\%28pp.\%2046\%E2\%80\%9368\%29.\%20Princeton \%3A\%2oPrinceton\%2oUniversity\%2oPress.)

Levy, J. K., Hipel, K. W., \& Howard, N. (2009a). Advances in drama theory for managing hazards and disasters. Part I: Theoretical foundation. Group Decision Negotiation, 18, 303-316.

CrossRef (https://doi.org/10.1007/s10726-008-9145-7)

Google Scholar (http://scholar.google.com

/scholar_lookup?title=Advances\%20in\%2odrama\%2otheory\%2ofor\%2omanagi ng\%2ohazards\%20and\%2odisasters.\%20Part\%2oI

\%3A\%2oTheoretical\%2ofoundation\&author=JK.\%20Levy\& author=KW.\%20Hipel\&author=N.\%20Howard\& journal=Group $\% 20$ Decision $\% 20$ Negotiation\&volume $=18 \&$ pages $=303-316 \&$ publication_year=2009)

Levy, J. K., Hipel, K. W., \& Howard, N. (2009b). Advances in drama theory for managing hazards and disasters. Part II: Coping with global climate change and environmental catastrophe. Group Decision Negotiation, 18, 317-334.

CrossRef (https://doi.org/10.1007/s10726-008-9144-8)

Google Scholar (http://scholar.google.com

/scholar_lookup?title=Advances\%20in\%20drama\%2otheory\%2ofor\%2omanagi ng\%2ohazards\%20and\%2odisasters.\%20Part\%2oII

\%3A\%2oCoping\%20with\%2oglobal\%20climate\%2ochange\%20and\%20environ mental\%20catastrophe\&author=JK.\%20Levy\&author=KW.\%20Hipel\& author=N.\%20Howard\&journal=Group\%2oDecision\%20Negotiation\& volume $=18 \&$ pages $=317-334 \&$ publication_year $=2009)$

Nasar, S. (1998). A beautiful mind. London: Faber and Faber.

Google Scholar (http://scholar.google.com/scholar_lookup?title=\%oA\%20 \%20\%20\%20\%20\%20\%20\%20\%20\%20\%20\%20\%20\%20\%20\%20\%20 \%20\%20\%20A\%2obeautiful\%20mind\%oA\%20\%20\%20\%20\%20\%20\%20 \%20\%20\%20\%20\%20\%20\%20\%20\%20\%20\%20\&author=S.\%20Nasar\& publication_year=1998)

Nash, J. (1950a). Equilibrium points in n-person games. PNAS: Proceedings of the National Academy of Sciences, 36, 48-49. In H. W. Khun (Ed.). (1996) Classics in game theory (pp. 3-4). Princeton: Princeton University Press. Google Scholar (https://scholar.google.com/scholar?q=Nash\%2C\%20J. \%20\%281950a\%29.\%20Equilibrium\%2opoints\%20in\%20nperson\%2ogames.\%2oPNAS

\%3A\%2oProceedings\%20of\%2othe\%2oNational\%2oAcademy\%20of\%2oScienc es\%2C\%2036\%2C\%2048\%E2\%80\%9349.\%20In\%20H.\%2oW.\%2oKhun \%20\%28Ed.\%29.\%20\%281996\%29\%20Classics\%20in\%2ogame\%2otheory \%20\%28pp.\%203\%E2\%80\%934\%29.\%2oPrinceton \%3A\%2oPrinceton\%2oUniversity\%2oPress.)

Nash, J. (1950b). The bargaining problem. Econometrica, 18, 155-162. In H. W. Khun (Ed.). (1996) Classics in game theory (pp. 5-13). Princeton: Princeton 
University Press.

Google Scholar (https://scholar.google.com/scholar?q=Nash\%2C\%20J. \%20\%281950b\%29.\%20The\%2obargaining\%20problem.\%20Econometrica \%2C\%2018\%2C\%20155\%E2\%80\%93162.\%20In\%2oH.\%2oW.\%2OKhun \%20\%28Ed.\%29.\%20\%281996\%29\%20Classics\%2oin\%2ogame\%2otheory \%20\%28pp.\%205\%E2\%80\%9313\%29.\%20Princeton \%3A\%2oPrinceton\%2oUniversity\%2oPress.)

Nash, J (1951) Non-cooperative games. Annals of Mathematics, 54, 286-295. In H. W. Khun (Ed.). (1996) Classics in game theory (pp. 14-26). Princeton: Princeton University Press.

Google Scholar (https://scholar.google.com/scholar?q=Nash\%2C\%20J \%20\%281951\%29\%20Noncooperative\%20games.\%20Annals\%20of\%2oMathematics\%2C\%2054 \%2C\%20286\%E2\%80\%93295.\%20In\%2OH.\%2oW.\%2OKhun\%20\%28Ed.\%29. \%20\%281996\%29\%20Classics\%20in\%2ogame\%2otheory\%20\%28pp.\%2014 \%E2\%80\%9326\%29.\%20Princeton\%3A\%20Princeton\%2oUniversity\%2oPress.)

Nordhaus, W. D. (2007). A review of the Stern review on the economics of climate change. Journal of Economic Literature, 45(3), 686-702.

CrossRef (https://doi.org/10.1257/jel.45.3.686)

Google Scholar (http://scholar.google.com

/scholar_lookup?title=A\%2oreview\%20of\%2othe\%2oStern\%2oreview\%20on\% 2othe\%20economics\%20of\%20climate\%20change\&

author=WD.\%20Nordhaus\&

journal=Journal\%20of $\%$ 20Economic $\% 2$ Literature\&volume $=45 \&$ issue $=3 \&$ pages $=686-702 \&$ publication_year=2007)

Nordhaus, W. (2015). Climate Clubs: Overcoming Free-Riding in International Climate Policy. American Economic Review, 105, 1339-1370.

CrossRef (https://doi.org/10.1257/aer.15000001)

Google Scholar (http://scholar.google.com

/scholar_lookup?title=Climate\%20Clubs\%3A\%20Overcoming\%2oFreeRiding\%20in\%2oInternational\%20Climate\%2oPolicy\& author=W.\%20Nordhaus\&journal=American\%20Economic\%2oReview\& volume $=105 \&$ pages $=1339-1370 \&$ publication_year=2015）

Pittel, K., \& Rübbelke, D. T. (2008). Climate policy and ancillary benefits: A survey and integration into the modelling of international negotiations on climate change. Ecological Economics, 68(1), 210-220.

Google Scholar (https://scholar.google.com/scholar?q=Pittel\%2C\%20K. \%2C\%20\%26\%20R\%C3\%BCbbelke\%2C\%20D.\%20T. \%20\%282008\%29.\%20Climate\%2opolicy\%20and\%20ancillary\%2obenefits \%3A\%20A\%20survey\%20and\%2ointegration\%2ointo\%2othe\%2omodelling\%2 oof\%2ointernational\%2onegotiations\%20on\%20climate\%2ochange.\%2oEcolog ical\%2oEconomics\%2C\%2068\%281\%29\%2C\%20210\%E2\%80\%93220.)

Pittel, K., \& Rübbelke, D. T. (2012). Transitions in the negotiations on climate change: from prisoner's dilemma to chicken and beyond. International Environmental Agreements: Politics, Law and Economics, 12(1), 23-39.

Google Scholar (https://scholar.google.com/scholar?q=Pittel\%2C\%20K. \%2C\%20\%26\%20R\%C3\%BCbbelke\%2C\%20D.\%20T.

\%20\%282012\%29.\%20Transitions\%20in\%2othe\%2onegotiations\%20on\%2ocli mate\%20change\%3A\%2ofrom\%2oprisoner\%E2\%80

\%99s\%20dilemma\%20to\%2ochicken\%20and\%2obeyond.\%2oInternational\%20 Environmental\%20Agreements\%3A\%20Politics

\%2C\%2oLaw\%2Oand\%2oEconomics\%2C\%2012\%281\%29\%2C\%2023\%E2 \%80\%9339.)

Robinson, D., \& Goforth, D. (2005). The topology of $2 x 2$ games: A new periodic table. New York: Routledge. 
Google Scholar (http://scholar.google.com/scholar_lookup?title=\%oA\%20 \%20\%20\%20\%20\%20\%20\%20\%20\%20\%20\%20\%20\%20\%20\%20\%20 \%20\%20\%20The\%2otopology\%20of\%202x2\%20games \%3A\%20A\%2onew\%2operiodic\%2otable\%oA\%20\%20\%20\%20\%20\%20\%20 \%20\%20\%20\%20\%20\%20\%20\%20\%20\%20\%20\&author=D.\%20Robinson\& author=D.\%20Goforth\&publication_year=2005)

Sachs, J. D. (2015). The age of sustainable development. New York, Columbia University Press.

Google Scholar (https://scholar.google.com/scholar?q=Sachs\%2C\%20J.\%20D. \%20\%282015\%29.\%20The\%20age\%20of\%2osustainable\%2odevelopment.\%20 New\%2oYork\%2C\%20Columbia\%20University\%2oPress.)

Sachs, J. (2005). The end of poverty: How we can make it happen in our lifetime. London: Penguin.

Google Scholar (http://scholar.google.com/scholar_lookup?title=\%oA\%20 \%20\%20\%20\%20\%20\%20\%20\%20\%20\%20\%20\%20\%20\%20\%20\%20 \%20\%20\%20The\%20end\%20of\%2opoverty \%3A\%20How\%20we\%20can\%2omake\%20it\%2ohappen\%2oin\%20our\%2olifeti me\%oA\%20\%20\%20\%20\%20\%20\%20\%20\%20\%20\%20\%20\%20\%20\%20 \%20\%20\%20\&author=J.\%20Sachs\&publication_year=2005)

Sen, A. K. (1999). Development as freedom. Alfred Knopf: New York. Google Scholar (http://scholar.google.com/scholar_lookup?title=\%oA\%20 \%20\%20\%20\%20\%20\%20\%20\%20\%20\%20\%20\%20\%20\%20\%20\%20 \%20\%20\%2oDevelopment\%20as\%2ofreedom\%oA\%20\%20\%20\%20\%20 \%20\%20\%20\%20\%20\%20\%20\%20\%20\%20\%20\%20\%20\& author=AK.\%20Sen\&publication_year=1999)

Shapley, L. (1953). A value for n-person games. In H. W. Kuhn, A. W. Tucker (Eds.), Contributions to the theory of games (Vol. II, pp. 307-317). In H. W. Kuhn (Ed.). (1996). Classics in game theory (pp. 69-79). Princeton: Princeton University Press.

Google Scholar (https://scholar.google.com/scholar?q=Shapley\%2C\%20L. \%20\%281953\%29.\%20A\%20value\%2ofor\%2onperson\%2Ogames.\%20In\%20H.\%2oW.\%20Kuhn\%2C\%2OA.\%2oW.\%2oTucker $\% 20 \% 28 \mathrm{Eds} . \% 29 \% 2 \mathrm{C}$

\%20Contributions\%20to\%2othe\%20theory\%20of\%2ogames\%20\%28Vol.\%20II \%2C\%20pp.\%20307\%E2\%80\%93317\%29.\%20In\%20H.\%20W.\%20Kuhn \%20\%28Ed.\%29.\%20\%281996\%29.\%20Classics\%20in\%2ogame\%2otheory \%20\%28pp.\%2069\%E2\%80\%9379\%29.\%20Princeton \%3A\%2oPrinceton\%2oUniversity\%2oPress.)

Soroos, M. S. (1994). Global change, environmental security, and the prisoner's dilemma. Journal of Peace Research, 31(3), 317-332.

CrossRef (https://doi.org/10.1177/0022343394031003006)

Google Scholar (http://scholar.google.com

/scholar_lookup?title=Global\%20change\%2C\%2oenvironmental\%20security \%2C\%20and\%20the\%2oprisoner\%E2\%80\%99s\%2odilemma\& author=MS.\%2oSoroos\&journal=Journal\%20of\%20Peace\%2oResearch\& volume $=31 \&$ issue $=3 \&$ pages $=317-332 \&$ publication_year $=1994 ）$

Stern, N. (2006). Stern review: The economics of climate change (Vol. 30). London: HM treasury.

Google Scholar (http://scholar.google.com /scholar_lookup?title=Stern\%2oreview \%3A\%20The\%20economics\%20of\%2oclimate\%2ochange\& author=N.\%20Stern\&publication_year=2006)

Stiglitz, J. E. (2002). Globalization and its Discontents (Vol. 500). New York: Norton.

Google Scholar (http://scholar.google.com/scholar_lookup?title=\%oA\%20 
\%20\%20\%20\%20\%20\%20\%20\%20\%20\%20\%20\%20\%20\%20\%20\%20 \%20\%20\%20Globalization\%20and\%20its\%20Discontents\%oA\%20\%20\%20 \%20\%20\%20\%20\%20\%20\%20\%20\%20\%20\%20\%20\%20\%20\%20\& author=JE.\%20Stiglitz\&publication_year=2002)

The Economist. (2007, September 27). Playing games with the planet. Accessed August 11, 2017, from http://www.economist.com/node/9867020 (http://www.economist.com/node/9867020)

UNDP. (2017). United Nations Human Development Report, NY. Google Scholar (https://scholar.google.com/scholar?q=UNDP. \%20\%282017\%29.\%20United\%2ONations\%20Human\%2oDevelopment\%2ORe port\%2C\%2ONY.)

Vajpeyi, D. K. (Ed.). (2013). Climate change, sustainable development and international security. Lexington (An imprint of Rowman \& Littlefield). isbn:978-0-7391-8146-1 https://rowman.com/ISBN/9780739181461 (https://rowman.com/ISBN/9780739181461)

Von Neumann, J. (1928). Zur Theorie der Gesellschaftsspiele. Mathematische Annalen, 100(1), 295-320. https://doi.org/10.1007/BFo1448847 (https://doi.org/10.1007/BF01448847)

Von Neumann, J., \& Morgenstern, O. (1944)[1994]. The theory of games and economic behavior. $50^{\text {th }}$ Anniversary Edition. Princeton, NJ: Princeton University Press.

Google Scholar (https://scholar.google.com/scholar?q=Von\%20Neumann \%2C\%20J.\%2C\%20\%26\%20Morgenstern\%2C\%20O.\%20\%281944 \%29\%5B1994\%5D.\%20The\%2otheory\%20of\%2ogames\%2oand\%2oeconomic\% 2obehavior.\%2050\%oAth\%20Anniversary\%2oEdition.\%2oPrinceton \%2C\%20NJ\%3A\%20Princeton\%20University\%20Press.)

Weber, M. (1978). In G. Roth \& C. Wittich (Eds.), Economy and society. Berkeley: University of California Press.

Google Scholar (http://scholar.google.com /scholar_lookup?\&author=M.\%2oWeber\&publication_year=1978)

Weitzman, M. L. (2007). The Stern review on the economics of climate change. Journal of Economic Literature, 45(3), 703-724.

CrossRef (https://doi.org/10.1257/jel.45.3.703)

Google Scholar (http://scholar.google.com

/scholar_lookup?title=The\%20Stern\%20review\%20on\%20the\%2oeconomics\% 20of\%2oclimate\%2ochange\&author=ML.\%2oWeitzman\& journal=Journal\%20of $\% 2$ Economic $\% 2$ Literature \&volume $=45 \&$ issue $=3 \&$ pages $=703-724 \&$ publication_year $=2007$ )

Wilensky, U. (2002). NetLogo PD Basic Evolutionary model. Evanston, IL: Center for Connected Learning and Computer-Based Modeling, Northwestern University http://ccl.northwestern.edu/netlogo/models/PDBasicEvolutionary (http://ccl.northwestern.edu/netlogo/models/PDBasicEvolutionary) Google Scholar (http://scholar.google.com/scholar_lookup?title=\%oA\%20 \%20\%20\%20\%20\%20\%20\%20\%20\%20\%20\%20\%20\%20\%20\%20\%20 \%20\%20\%20NetLogo\%20PD\%20Basic\%20Evolutionary\%2omodel\%oA\%20 \%20\%20\%20\%20\%20\%20\%20\%20\%20\%20\%20\%20\%20\%20\%20\%20\%20\& author=U.\%20Wilensky\&publication_year=2002)

\section{Copyright information}

\section{(C) Springer Nature Switzerland AG 2019}




\section{About this chapter}

Cite this chapter as:

Caleiro A.B., de Sousa M.R., de Oliveira I.A. (2019) Global Development and Climate Change: A Game Theory Approach. In: Sequeira T., Reis L. (eds) Climate Change and Global Development. Contributions to Economics. Springer, Cham

\section{First Online}

09 May 2019

\section{DOI}

https://doi.org/10.1007/978-3-030-02662-2_2

\section{Publisher Name}

Springer, Cham

\section{Print ISBN}

978-3-030-02661-5

\section{Online ISBN}

978-3-030-02662-2

\section{eBook Packages}

Economics and Finance

Buy this book on publisher's site

Reprints and Permissions

\section{SPRINGER NATURE}

(C) 2018 Springer Nature Switzerland AG. Part of Springer Nature. Not logged in · Not affiliated · 85.243.186.224 